\title{
Single-site-resolved measurement of the current statistics in optical lattices
}

\author{
Stefan Keßler ${ }^{1}$ and Florian Marquardt ${ }^{1,2}$ \\ ${ }^{1}$ Institute for Theoretical Physics, Universität Erlangen-Nürnberg, Staudtstraße 7, 91058 Erlangen, Germany \\ ${ }^{2}$ Max Planck Institute for the Science of Light, Günther-Scharowsky-Straße 1/Bau 24, 91058 Erlangen, Germany
}

(Received 11 October 2013; published 3 June 2014)

\begin{abstract}
At present, great effort is spent on the experimental realization of gauge fields for quantum many-body systems in optical lattices. At the same time, the single-site-resolved detection of individual atoms has become a new powerful experimental tool. We discuss a protocol for the single-site-resolved measurement of the current statistics of quantum many-body systems, which makes use of a bichromatic optical superlattice and single-site detection. We illustrate the protocol by a numerical study of the current statistics for interacting bosons in one and two dimensions and discuss the role of the on-site interactions for the current pattern and the ground-state symmetry for small two-dimensional lattices with artificial magnetic fields.
\end{abstract}

DOI: 10.1103/PhysRevA.89.061601

PACS number(s): 67.85.-d, 03.65.Vf, 05.60.Gg

Introduction. Improved and new detection techniques have contributed significantly to the rapid progress in the study of strongly correlated states of ultracold atoms in optical lattices in recent years. The combination of a time-of-flight expansion followed by absorption imaging gives access to the momentum distribution and has revealed the superfluid-toMott-insulator transition [1]. Going further, one can study the noise correlations in these absorption images, e.g., exhibiting the quantum statistics of bosons [2] and fermions [3]. In addition, the excitation spectrum of interacting bosons has been measured using momentum-resolved Bragg spectroscopy [4]. However, the most significant step in recent times has arguably been the introduction of single-site detection (using fluorescence microscopy) in optical lattices [5,6]. This new method has been used to observe the shell structure of a Mott insulator [6,7], the propagation of single bosons [8], a spin impurity [9], and the structure of density correlations [10]. In the future, it may provide new insights into the buildup of entanglement in many-body systems and the influence of measurements on quantum many-body dynamics [11-13].

Here, we discuss a scheme for the spatially resolved measurement of the current between nearest-neighbor lattice sites. It relies on combining a bichromatic superlattice and single-site detection. Such a tool is especially timely in the context of the recent effort towards realizing gauge fields in optical lattices (for recent reviews, see [14-16]), with first implementations reported in [17-21]. These systems can be used to realize states that support equilibrium currents. Various methods have by now been proposed to detect interesting aspects of such systems, e.g., quantum Hall edge states and Chern numbers in topological insulators, using time-of-flight expansion [22-26], light scattering [27,28], or the time evolution of the real-space density after a quench in the potential [29-31]. The protocol discussed below has similarities with the latter approach. However, in addition to probing the current pattern [29], it reveals the full spatially resolved current statistics of the quantum many-body state, from which correlation functions can be extracted.

Model and current operator. We consider a tight-binding Hamiltonian for ultracold atoms in an optical lattice,

$$
\hat{\mathcal{H}}=-\sum_{\langle l, r\rangle}\left\{J_{l r} \hat{c}_{l}^{\dagger} \hat{c}_{r}+J_{l r}^{*} \hat{c}_{r}^{\dagger} \hat{c}_{l}\right\}+\sum_{i} \epsilon_{i} \hat{n}_{i}+\hat{\mathcal{H}}_{\text {int }} .
$$

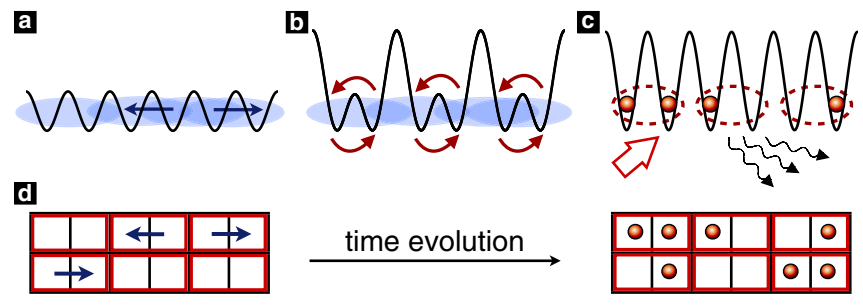

FIG. 1. (Color online) (a)-(c) Protocol for measuring the current statistics of a quantum many-body state in an optical lattice. (b) An additional optical lattice with double wavelength is suddenly ramped up [for a two-dimensional (2D) system, tunneling in the other direction is also turned off] and the on-site interaction between atoms is set close to zero. Afterwards, the atoms move within a double-well potential. (c) After some time, the motion is frozen out completely by also ramping up the barrier within each double well, and the atoms are detected by a single-site-resolved measurement. (d) An appropriate choice [see Eq. (3)] of the evolution time in the double-well potential (b) maps the current operator to the difference of the particle number at the right and left lattice site (shown for a 2D setup) and realizes a spatially resolved measurement of the current operator [Eq. (2)].

Here, $\hat{c}_{l}^{(\dagger)}$ is a bosonic or fermionic annihilation (creation) operator, $J_{l r}$ is the possibly complex tunneling amplitude, and $\langle$,$\rangle denotes pairs of nearest-neighbor lattice sites. The$ interaction part $\hat{\mathcal{H}}_{\text {int }}$ is a polynomial of local particle number operators, $\hat{n}_{i}=\hat{c}_{i}^{\dagger} \hat{c}_{i}$, and $\epsilon_{i}$ is the on-site energy at site $i$. The (mass) current operator for this system can be defined using the local continuity equation for the particle density. For a lattice system, it reads $\frac{d}{d t} \hat{n}_{l}+\sum_{r \in \mathrm{NN}(l)} \hat{J}_{l \rightarrow r}=0$, where $\hat{J}_{l \rightarrow r}$ denotes the current from site $l$ to site $r$ and $\mathrm{NN}(l)$ denotes the set of nearest-neighbor lattice sites of $l$. Using Heisenberg's equation of motion gives $\frac{d}{d t} \hat{n}_{l}=i \sum_{r \in \mathrm{NN}(l)}\left\{J_{l r} \hat{c}_{l}^{\dagger} \hat{c}_{r}-\right.$ $\left.J_{l r}^{*} \hat{c}_{r}^{\dagger} \hat{c}_{l}\right\}$, and accordingly the current operator between nearestneighbor lattice sites $l$ and $r$ reads (for bosons and fermions)

$$
\hat{J}_{l \rightarrow r}=-i\left\{J_{l r} \hat{c}_{l}^{\dagger} \hat{c}_{r}-J_{l r}^{*} \hat{c}_{r}^{\dagger} \hat{c}_{l}\right\}
$$

Measurement protocol. The protocol for measuring the eigenvalues of the current operator (2) is summarized in Fig. 1. The main idea is to use a bichromatic superlattice, which has already been realized experimentally $[32,33]$, and 
to apply a beam-splitter operation to map the single-particle eigenstates of the current operator to the states localized at the left and right lattice site of each double well [Fig. 1(b)]. The measured value of the current operator is then essentially given by the difference of the particle numbers of both lattice sites. The particle number can be measured [Figs. 1(c) and $1(\mathrm{~d})$ ] in principle by the recently developed single-site imaging techniques [5,6], although at present these are still restricted to parity measurements (see the discussion on experimental details below).

Formally, the protocol relies on the time evolution of noninteracting atoms in a symmetric double-well potential, $\hat{\mathcal{H}}=-\left(J_{l r} \hat{c}_{l}^{\dagger} \hat{c}_{r}+J_{l r}^{*} \hat{c}_{r}^{\dagger} \hat{c}_{l}\right)$. The difference in the atom number at the two wells oscillates in time and can be expressed as

$$
\hat{n}_{r}(t)-\hat{n}_{l}(t)=\cos (2 J t)\left[\hat{n}_{r}(0)-\hat{n}_{l}(0)\right]+\sin (2 J t) \frac{\hat{J}_{l \rightarrow r}(0)}{J},
$$

where $J=\left|J_{l r}\right|$. Thus, the current can be obtained as the density difference, $\hat{\jmath}_{l \rightarrow r}(0)=(-1)^{m} J\left[\hat{n}_{r}(\tilde{t})-\hat{n}_{l}(\tilde{t})\right]$, for suitable chosen evolution times $J \tilde{t}=\pi(2 m+1) / 4, m \in \mathbb{N}_{0}$.

The previous expression for $\hat{\jmath}_{l \rightarrow r}(0)$ shows that the current operator has discrete eigenvalues, just like the particle number operator. This situation, surprising at first sight, can also be understood as follows: The eigenvalues of the current operator are given by the difference in the density of atoms going to the right and left times the velocity. This is seen by diagonalizing the current operator (2), which yields $\hat{J}_{l \rightarrow r}=$ $J\left(\hat{c}_{\rightarrow}^{\dagger} \hat{c}_{\rightarrow}-\hat{c}_{\leftarrow}^{\dagger} \hat{c}_{\leftarrow}\right)$ with $\hat{c}_{\rightarrow}=\left(\hat{c}_{r}+i J_{l r}^{*} \hat{c}_{l} / J\right) / \sqrt{2}$ and $\hat{c}_{\leftarrow}=$ $\left(\hat{c}_{r}-i J_{l r}^{*} \hat{c}_{l} / J\right) / \sqrt{2}$. The operators $\hat{c}_{\rightarrow}$ and $\hat{c}_{\leftarrow}$ have a simple meaning: They correspond to right- and left-going atoms [34]. Since the total particle number in the double well, $\hat{n}_{l}+\hat{n}_{r}$, commutes with $\hat{J}_{l \rightarrow r}$, we can assume a situation of fixed $n_{l}+n_{r}$. Then the spectrum of the current operator is $J \cdot\{-n,-n+2, \ldots, n\}$, with $n=n_{l}+n_{r}$ for bosons and $n=\left[n_{l}+n_{r}\right] \bmod 2$ for fermions due to the Pauli principle.

Bosons in 1D. We first consider the current statistics of the homogeneous one-dimensional (1D) Bose-Hubbard model,

$$
\hat{\mathcal{H}}=-J \sum_{i}\left\{\hat{c}_{i+1}^{\dagger} \hat{c}_{i}+\hat{c}_{i}^{\dagger} \hat{c}_{i+1}\right\}+\frac{U}{2} \sum_{i} \hat{n}_{i}\left(\hat{n}_{i}-1\right),
$$

with real tunneling amplitude $J$ and on-site interaction strength $U$. To keep the numerics manageable, we focus on the $1 \mathrm{D}$ case, even though we believe that the qualitative features of the local properties we are going to discuss should not be dependent on dimensionality in a significant way. The two phases of the Bose-Hubbard model exhibit a characteristic atom number statistics at a single site: a Poisson distribution for the superfluid state $(U / J \rightarrow 0)$ and a fixed atom number in the Mott insulating regime $(U / J \rightarrow \infty)$ for integer filling $\bar{n}$; see, e.g., Refs. [35,36].

The corresponding current statistics is presented in Figs. 2(a)-2(d) at filling $\bar{n}=1$. For $U=0$, the atoms are in a product state of coherent states at the individual sites. Thus, $n_{\rightarrow}$ and $n_{\leftarrow}$ are also Poisson distributed, with mean $\bar{n}$. The current (2), being the difference between two Poisson distributed variables, is then given by the so-called Skellam distribution, $P_{\bar{n}}(j=J m)=e^{-2 \bar{n}} \mathcal{I}_{|m|}(2 \bar{n})$, where $\mathcal{I}$ denotes the modified Bessel function. When increasing $U / J$, the distribution becomes more and more concentrated at the
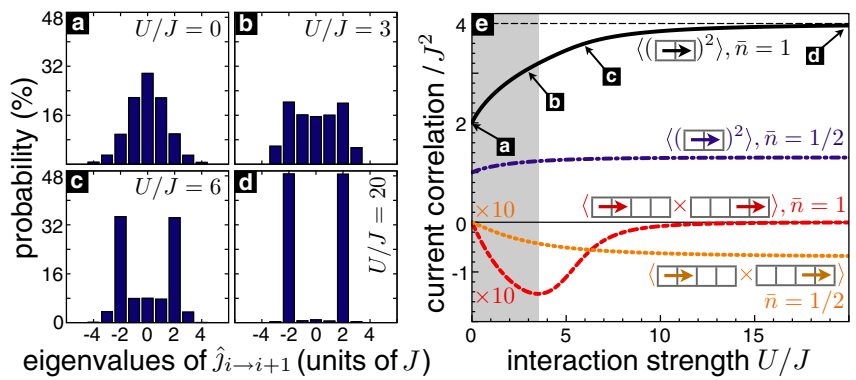

FIG. 2. (Color online) Current statistics for the ground state of interacting bosons in a 1D lattice. The results are obtained by exact diagonalization of a lattice with 12 or 16 sites for filling $\bar{n}=1$ and $\bar{n}=1 / 2$, respectively, and periodic boundary conditions. (a)-(d) Distribution of the current eigenvalues for $\bar{n}=1$ and interaction strength $U / J=0,3,6,20$ [(a)-(d)] calculated from 25000 snapshots. The vanishing mean current $\left\langle\hat{\jmath}_{i \rightarrow i+1}\right\rangle$ is reflected by the $j \mapsto-j$ symmetry of the distributions. (e) Interaction dependence of the variance of the current, $\left\langle\left(\hat{\jmath}_{i \rightarrow i+1}\right)^{2}\right\rangle$, and the current-current correlation $\left\langle\hat{J}_{i \rightarrow i+1} \hat{J}_{i+2 \rightarrow i+3}\right\rangle$ for unit filling and half filling. The variance of the current increases monotonically with $U / J$ in both cases. Interestingly, extremal values of the current-current correlation show up at intermediate interaction strengths for $\bar{n}=1$.

eigenvalues $\pm 2 J$. This is a consequence of the Mott insulating state being a superposition of the eigenstates corresponding to $j= \pm 2 J, \hat{c}_{i}^{\dagger} \hat{c}_{i+1}^{\dagger}|\mathrm{vac}\rangle=\frac{1}{2}\left[\left(\hat{c}_{\rightarrow}^{\dagger}\right)^{2}-\left(\hat{c}_{\leftarrow}^{\dagger}\right)^{2}\right]|\mathrm{vac}\rangle$. Note that in general, the current eigenvalues are even multiples of $J$ for the Mott insulator at arbitrary integer filling.

Figure 2(e) shows the interaction dependence of current correlation functions, which might be detected using the scheme proposed here. The variance of the current increases monotonically with the on-site interaction strength, from $\left\langle\hat{\jmath}_{i \rightarrow i+1}^{2}\right\rangle_{\mathrm{SF}}=2 J^{2} \bar{n}$ for $U=0$ to $\left\langle\hat{\jmath}_{i \rightarrow i+1}^{2}\right\rangle_{\mathrm{MI}}=2 J^{2} \bar{n}(\bar{n}+1)$ at integer filling $\bar{n}$ deep in the Mott insulating regime. For $\bar{n}=1$, the current correlation between neighboring pairs of lattice sites, $\left\langle\hat{J}_{i \rightarrow i+1} \hat{J}_{i+2 \rightarrow i+3}\right\rangle$, becomes negative for intermediate $U / J$ with a minimum close to the superfluid-to-Mott-insulator transition, while it vanishes for $U \rightarrow 0$ and $U / J \rightarrow \infty$. In contrast, for a half-filled lattice, $\left\langle\hat{J}_{i \rightarrow i+1} \hat{J}_{i+2 \rightarrow i+3}\right\rangle$ decreases asymptotically as one goes into the hardcore boson limit $(U / J \rightarrow \infty)$.

We note that the correlations of the current into and through a lattice site might be accessed by an extended version of the measurement scheme using a triple-well superlattice structure; see Supplemental Material [37].

Bosons in a synthetic magnetic field. We consider interacting bosons subject to a uniform synthetic magnetic field perpendicular to the 2D lattice. The corresponding Hamiltonian reads, in Landau gauge,

$$
\begin{aligned}
\hat{\mathcal{H}}= & -J \sum_{x, y}\left\{\hat{c}_{x+1, y}^{\dagger} \hat{c}_{x, y}+\hat{c}_{x, y+1}^{\dagger} \hat{c}_{x, y} e^{i 2 \pi \alpha x}+\text { H.c. }\right\} \\
& +\frac{U}{2} \sum_{x, y} \hat{n}_{x, y}\left(\hat{n}_{x, y}-1\right) .
\end{aligned}
$$

Here, $x, y$ are the integer $x$ and $y$ coordinates of the lattice sites, and the phase $2 \pi \alpha$, which a boson picks up when circulating in an anticlockwise direction around a unit cell, encodes the 

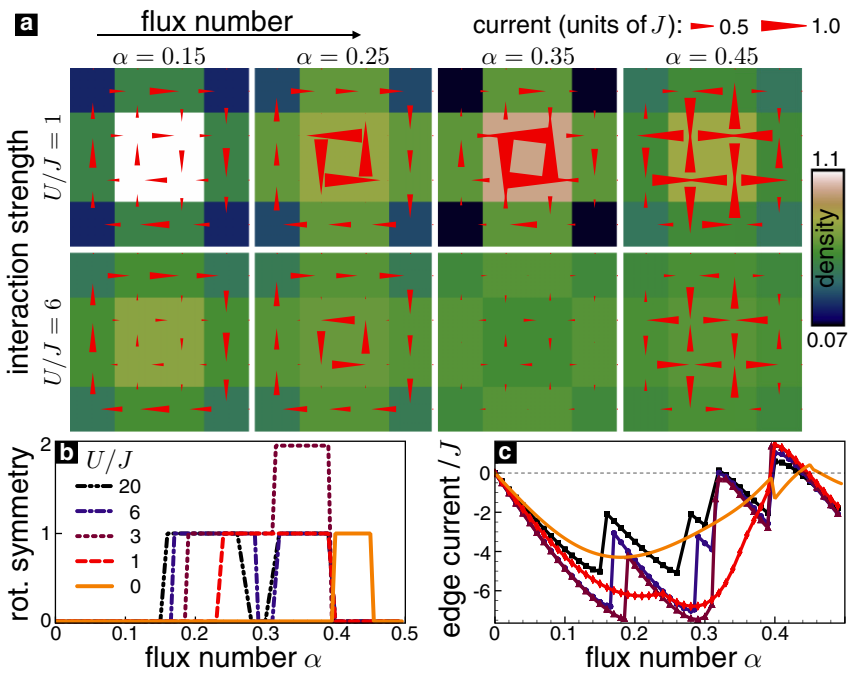

FIG. 3. (Color online) Ground-state properties of nine interacting bosons in a $4 \times 4$ lattice with artificial magnetic field (similar behavior is found for different fillings): (a) current and density pattern, (b) rotational symmetry, and (c) edge current. Increasing the flux number starting from $\alpha=0$, the current flows in clockwise directions and grows with $\alpha$. The current in the center is generally suppressed for larger $U / J$, and it reverses its direction above a certain critical value of $\alpha$ (which decreases with increasing $U / J$ ). This change appears together with (b) a transition in the rotational symmetry of the ground state and (c) a discontinuity in the edge current. Large interactions lead to additional configurations, as shown, e.g., in the panel for $U / J=6$ and $\alpha=0.35$.

effect of the magnetic field. For a charged particle, $\alpha$ equals the number of flux quanta per unit cell. The single-particle spectrum of (5) is given by the famous fractal "Hofstadter butterfly" [38].

Below we discuss relatively small 2D lattices, which might be realized first in experiments (with a suitable superlattice structure dividing the entire lattice into such small plaquettes as implemented for $2 \times 2$ lattices in [17]). A similar system has been realized with Bose-Einstein condensates in rotating lattices $[39,40]$, where the "Lorentz force" is replaced by the Coriolis force [41]. The creation and observation of topological states in this setup have been theoretically studied in [42-47]. Moreover, a transition between ground states of different rotational symmetry at discrete rotation frequencies was found $[48,49]$, which leads to a discontinuity in the edge current. Here, we study the effects of finite on-site interactions on such transitions (these previous works $[48,49]$ discussed only the limit of hardcore bosons).

The results obtained from exact diagonalization for a $4 \times 4$ lattice are summarized in Fig. 3. Note that we restrict ourselves to the interval $\alpha \in[0,0.5]$, as the Hamiltonian is invariant under $\alpha \mapsto \alpha+1$, and $\alpha \mapsto-\alpha$ only changes the magnetic field direction. Figure 3(a) shows the current and density profile of the ground state for different interaction strengths and $\alpha$ 's. The current patterns found for $U / J=1$ are similar to those of $U / J=0$ (not shown), whereas additional current configurations appear for large $U / J$. Note that the central current reverses sign beyond some critical flux number $\alpha$, and this value decreases for larger $U / J$.
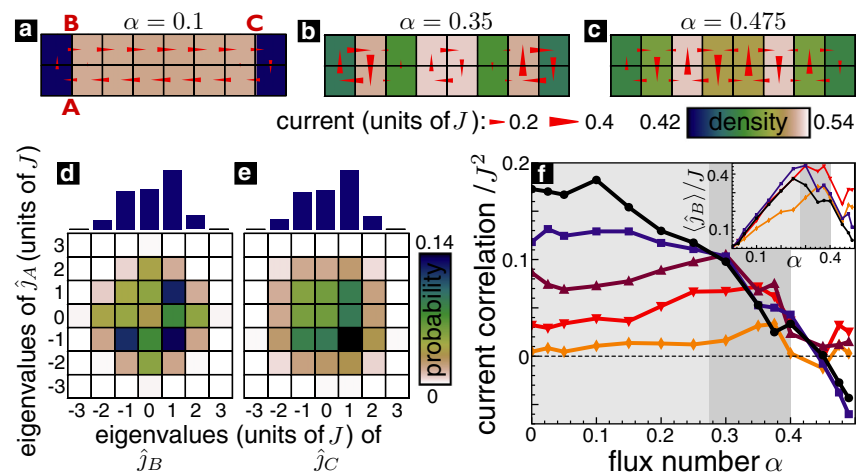

FIG. 4. (Color online) Current statistics of the ground state of interacting bosons in a $8 \times 2$ lattice at half filling, calculated from 25000 snapshots. (a)-(c) Density and current profile for interaction strength $U / J=8$ and different flux numbers $\alpha$. (d),(e) Joint eigenvalue distribution for different current operators and the parameters used in (a). Note that the currents at the links A, B, and C are defined in the positive $x$ direction. While $\hat{j}_{B}$ and $\hat{\jmath}_{C}$ have the same eigenvalue distribution (see histograms), their joint distribution with $\hat{J}_{A}$ is different: $\hat{\jmath}_{A}$ and $\hat{J}_{C}$ are uncorrelated, but $\hat{J}_{A}$ and $\hat{\jmath}_{B}$ are correlated. (f) Current correlation $\left\langle\hat{\jmath}_{A} \hat{\jmath}_{B}\right\rangle-\left\langle\hat{\jmath}_{A}\right\rangle\left\langle\hat{\jmath}_{B}\right\rangle$ for different interaction strengths $(U / J=0.5,2,4,8,25$, bright to dark). Surprisingly, large on-site interactions lead to a strong positive current correlation for flux numbers corresponding to the current pattern (a). The inset shows the $\alpha$ dependence of the mean current $\left\langle\hat{j}_{B}\right\rangle$ for the same interaction strengths (note that $\left\langle\hat{j}_{A}\right\rangle=-\left\langle\hat{j}_{B}\right\rangle$ ).

The critical value of $\alpha$ can be identified via the change in the ground-state rotational symmetry or via the first discontinuity of the edge current. We define the edge current as the sum of all currents along the boundary, counted in an anticlockwise direction; see Fig. 3(c). The rotational symmetry is best discussed in the symmetric gauge; see [37]. For a square lattice, the Hamiltonian (5) commutes with the rotation by $\pi / 2$, $\mathcal{R}(\pi / 2)$. Thus, a nondegenerate ground state is an eigenstate of $\mathcal{R}(\pi / 2)$, with eigenvalue $e^{i \pi m / 2}, m=0,1,2,3$. Additional transitions in the ground-state symmetry (discontinuities of the edge current) show up for intermediate $U / J$. The critical flux numbers for these transition points hardly change for $U / J \gtrsim 10$. The discussed current patterns and the edge current can be measured using the proposed protocol, which therefore provides a means of studying the flux and interaction dependence of such transitions.

We now turn to spatial current correlations. An illustrative example is displayed in Fig. 4, for a half-filled $8 \times 2$ lattice. Such bosonic flux ladders exhibit a transition from a Meissner phase [Fig. 4(a)] to a vortex phase [Fig. 4(b)] when $\alpha$ is increased [50,51]. For the situation shown in Fig. 4(a), we address the question of whether the currents at A, B, and $\mathrm{C}$ are correlated. This is done by constructing the joint probability distribution of the eigenvalues from an ensemble of snapshots, since the currents $\hat{J}_{A}, \hat{\jmath}_{B}$, and $\hat{J}_{C}$ can be measured simultaneously. Figures 4(d) and 4(e) display two examples of these joint probability distributions. The measured values of $\hat{J}_{A}$ and $\hat{J}_{C}$ at the two far-removed links $\mathrm{A}$ and $\mathrm{C}$ are (to a very good approximation) independent of each other, i.e., the joint probability distribution is just a product of the eigenvalue distribution of $\hat{J}_{A}$ and $\hat{J}_{C}$ [cf. Fig. 4(e)]. In contrast, the current 
operators $\hat{J}_{A}$ and $\hat{J}_{B}$ for nearby sites are correlated as the joint probabilities $p\left(j_{A}=-J, j_{B}=-J\right)$ and $p\left(j_{A}=-J, j_{B}=0\right)$ are clearly different even though $p\left(j_{B}=-J\right)$ and $p\left(j_{B}=0\right)$ are almost equal; see Fig. 4(d). The dependence of the current correlation $\left\langle\hat{j}_{A} \hat{\jmath}_{B}\right\rangle-\left\langle\hat{j}_{A}\right\rangle\left\langle\hat{j}_{B}\right\rangle$ on the flux number $\alpha$ is shown in Fig. 4(f). We find a positive correlation for $\alpha \lesssim 0.3$ [parameter regime with the current pattern shown in Fig. 4(a)], which becomes stronger with increasing on-site interaction strength. In contrast, the average current [inset in Fig. 4(f)] hardly changes with the interaction strength for $U / J \gtrsim 2$. For larger flux values, the correlation falls off to values around zero.

Discussion of experimental details. Let us consider the combination of the proposed measurement protocol with gauge fields created by laser-assisted tunneling [52-54] implemented in $[17,21,55]$. The required 2D lattice consists of alternating columns with different on-site energies (and may trap different internal states [52,53]). Tunneling between different columns is only nonzero when it is driven by additional light fields (which also imprint the phase on the tunneling amplitude), while bare tunneling exists within each column. Thus, the bichromatic superlattice for the current measurement could be applied in the direction of the columns, while the tunneling between the columns is inhibited by switching off the driving laser fields.

All of the present experiments on single-site-resolved detection only resolve the parity of the atom number at any lattice site $[5,6]$. For a single or two coupled 1D chains, considered in Figs. 2 and 4, one might let the atoms expand into another direction before the detection process (similar to [8]) to avoid double or higher occupancies. However, for true 2D configurations, one is currently restricted to small filling factors, until the parity problem can be circumvented via alternative approaches. We have further investigated these limitations of the measurement protocol for the configuration shown in Fig. 4, by numerically simulating situations with parity detection only. We also included residual interactions, $U_{\text {res }}$, and timing errors during the evolution in the double-well potential. We find that the current pattern and the current correlation can still be observed in the presence of parity detection, even though the absolute value of the observed current decreases by up to $25 \%$. The influence of the residual interaction is of the order of a few percent for $U_{\text {res }} / J \leqslant 1 / 4$. A timing error $J \tilde{t}=\pi / 4+J \Delta t$ leads to a change in the current and current correlation of less than $0.02 \mathrm{~J}$ and $0.01 \mathrm{~J}^{2}$, respectively, for $J \Delta t \leqslant 0.05$.

Conclusions. We have analyzed a protocol for the siteresolved measurement of the current operator in optical lattices. Using already available experimental techniques, it can be employed for interacting bosons at small filling factors. It can, in principle, be extended to fermions and possibly also to situations with different species. Measuring the statistics and spatial structure of currents seems a promising tool to study the physics of interacting ultracold atoms subject to gauge fields.

Note added. The Meissner phase in a bosonic flux ladder [cf. Fig. 4(a)] was recently observed experimentally by measuring the average edge currents [56].

Acknowledgments. We thank the DFG for support in the Emmy-Noether programme and the SFB/TR 12.
[1] M. Greiner, O. Mandel, T. Esslinger, T. W. Hänsch, and I. Bloch, Nature (London) 415, 39 (2002).

[2] S. Fölling, F. Gerbier, A. Widera, O. Mandel, T. Gericke, and I. Bloch, Nature (London) 434, 481 (2005).

[3] T. Rom, T. Best, D. van Oosten, U. Schneider, S. Fölling, B. Paredes, and I. Bloch, Nature (London) 444, 733 (2006).

[4] P. T. Ernst, S. Götze, J. S. Krauser, K. Pyka, D.-S. Lühmann, D. Pfannkuche, and K. Sengstock, Nat. Phys. 6, 56 (2010).

[5] W. S. Bakr, J. I. Gillen, A. Peng, S. Fölling, and M. Greiner, Nature (London) 462, 74 (2009).

[6] J. F. Sherson, C. Weitenberg, M. Endres, M. Cheneau, I. Bloch, and S. Kuhr, Nature (London) 467, 68 (2010).

[7] W. S. Bakr, A. Peng, M. E. Tai, R. Ma, J. Simon, J. I. Gillen, S. Fölling, L. Pollet, and M. Greiner, Science 329, 547 (2010).

[8] C. Weitenberg, M. Endres, J. F. Sherson, M. Cheneau, P. Schauß, T. Fukuhara, I. Bloch, and S. Kuhr, Nature (London) 471, 319 (2011).

[9] T. Fukuhara, A. Kantian, M. Endres, M. Cheneau, P. Schauß, S. Hild, D. Bellem, U. Schollwöck, T. Giamarchi, C. Gross, I. Bloch, and S. Kuhr, Nat. Phys. 9, 235 (2013).

[10] M. Endres, M. Cheneau, T. Fukuhara, C. Weitenberg, P. Schauß, C. Gross, L. Mazza, M. Bañuls, L. Pollet, I. Bloch, and S. Kuhr, Science 334, 200 (2011).

[11] A. J. Daley, H. Pichler, J. Schachenmayer, and P. Zoller, Phys. Rev. Lett. 109, 020505 (2012).
[12] S. Keßler, I. P. McCulloch, and F. Marquardt, New J. Phys. 15, 053043 (2013).

[13] S. Keßler, A. Holzner, I. P. McCulloch, J. von Delft, and F. Marquardt, Phys. Rev. A 85, 011605(R) (2012).

[14] J. Dalibard, F. Gerbier, G. Juzeliūnas, and P. Öhberg, Rev. Mod. Phys. 83, 1523 (2011).

[15] M. Lewenstein, A. Sanpera, and V. Ahufinger, Ultracold Atoms in Optical Lattices: Simulating Quantum Many-body Systems, 1st ed. (Oxford University Press, Oxford, 2012).

[16] N. Goldman, G. Juzeliūnas, P. Öhberg, and I. B. Spielman, arXiv:1308.6533.

[17] M. Aidelsburger, M. Atala, S. Nascimbène, S. Trotzky, Y.-A. Chen, and I. Bloch, Phys. Rev. Lett. 107, 255301 (2011).

[18] J. Struck, C. Ölschläger, M. Weinberg, P. Hauke, J. Simonet, A. Eckardt, M. Lewenstein, K. Sengstock, and P. Windpassinger, Phys. Rev. Lett. 108, 225304 (2012).

[19] K. Jiménez-García, L. J. LeBlanc, R. A. Williams, M. C. Beeler, A. R. Perry, and I. B. Spielman, Phys. Rev. Lett. 108, 225303 (2012).

[20] M. Aidelsburger, M. Atala, M. Lohse, J. T. Barreiro, B. Paredes, and I. Bloch, Phys. Rev. Lett. 111, 185301 (2013).

[21] H. Miyake, G. A. Siviloglou, C. J. Kennedy, W. C. Burton, and W. Ketterle, Phys. Rev. Lett. 111, 185302 (2013).

[22] V. W. Scarola and S. Das Sarma, Phys. Rev. Lett. 98, 210403 (2007). 
[23] E. Alba, X. Fernandez-Gonzalvo, J. Mur-Petit, J. K. Pachos, and J. J. Garcia-Ripoll, Phys. Rev. Lett. 107, 235301 (2011).

[24] E. Zhao, N. Bray-Ali, C. J. Williams, I. B. Spielman, and I. I. Satija, Phys. Rev. A 84, 063629 (2011).

[25] T. P. Polak and T. A. Zaleski, Phys. Rev. A 87, 033614 (2013).

[26] L. Wang, A. A. Soluyanov, and M. Troyer, Phys. Rev. Lett. 110, 166802 (2013).

[27] J. S. Douglas and K. Burnett, Phys. Rev. A 84, 053608 (2011).

[28] N. Goldman, J. Beugnon, and F. Gerbier, Phys. Rev. Lett. 108, 255303 (2012).

[29] M. Killi and A. Paramekanti, Phys. Rev. A 85, 061606(R) (2012).

[30] M. Killi, S. Trotzky, and A. Paramekanti, Phys. Rev. A 86, 063632 (2012).

[31] N. Goldman, J. Dalibard, A. Dauphin, F. Gerbier, M. Lewenstein, P. Zoller, and I. B. Spielman, Proc. Natl. Acad. Sci. 110, 6736 (2013).

[32] J. Sebby-Strabley, M. Anderlini, P. S. Jessen, and J. V. Porto, Phys. Rev. A 73, 033605 (2006).

[33] S. Trotzky, Y.-A. Chen, U. Schnorrberger, P. Cheinet, and I. Bloch, Phys. Rev. Lett. 105, 265303 (2010).

[34] More precisely, if we were to consider an extended 1D tightbinding lattice with nearest-neighbor tunneling amplitude $J_{l r}$, these would be the states with maximal velocity $\pm 2 J$, at the momenta $k= \pm \pi / 2-\arg \left(J_{l r}\right)$.

[35] D. Jaksch, C. Bruder, J. I. Cirac, C. W. Gardiner, and P. Zoller, Phys. Rev. Lett. 81, 3108 (1998).

[36] I. Bloch, J. Dalibard, and W. Zwerger, Rev. Mod. Phys. 80, 885 (2008).

[37] See Supplemental Material at http://link.aps.org/supplemental/ 10.1103/PhysRevA.89.061601 for the discussion of an extended version of the measurement scheme, the rotational symmetry, and the effect of timing errors.
[38] D. R. Hofstadter, Phys. Rev. B 14, 2239 (1976).

[39] S. Tung, V. Schweikhard, and E. A. Cornell, Phys. Rev. Lett. 97, 240402 (2006).

[40] R. A. Williams, S. Al-Assam, and C. J. Foot, Phys. Rev. Lett. 104, 050404 (2010).

[41] N. R. Cooper, Adv. Phys. 57, 539 (2008).

[42] N. Barberán, M. Lewenstein, K. Osterloh, and D. Dagnino, Phys. Rev. A 73, 063623 (2006).

[43] M. Hafezi, A. S. Sørensen, E. Demler, and M. D. Lukin, Phys. Rev. A 76, 023613 (2007).

[44] R. N. Palmer, A. Klein, and D. Jaksch, Phys. Rev. A 78, 013609 (2008).

[45] R. O. Umucalilar, H. Zhai, and M. O. Oktel, Phys. Rev. Lett. 100, 070402 (2008).

[46] R. O. Umucalilar and E. J. Mueller, Phys. Rev. A 81, 053628 (2010).

[47] T. Graß, B. Juliá-Díaz, and M. Lewenstein, Phys. Rev. A 86, 053629 (2012).

[48] R. Bhat, M. J. Holland, and L. D. Carr, Phys. Rev. Lett. 96, 060405 (2006).

[49] R. Bhat, B. M. Peden, B. T. Seaman, M. Krämer, L. D. Carr, and M. J. Holland, Phys. Rev. A 74, 063606 (2006).

[50] E. Orignac and T. Giamarchi, Phys. Rev. B 64, 144515 (2001).

[51] M.-C. Cha and J.-G. Shin, Phys. Rev. A 83, 055602 (2011).

[52] D. Jaksch and P. Zoller, New J. Phys. 5, 56 (2003).

[53] F. Gerbier and J. Dalibard, New J. Phys. 12, 033007 (2010).

[54] A. R. Kolovsky, Europhys. Lett. 93, 20003 (2011).

[55] M. Aidelsburger, M. Atala, S. Nascimbène, S. Trotzky, Y.-A. Chen, and I. Bloch, Appl. Phys. B 113, 1 (2013).

[56] M. Atala, M. Aidelsburger, M. Lohse, J. T. Barreiro, B. Paredes, and I. Bloch, arXiv:1402.0819. 